\title{
EXTRAÇÃO DO ÓLEO DAS SEMENTES DE CRAMBE (Crambe abyssinica) EMPREGANDO PROPANO SUBCRÍTICO
}

\author{
K. A. SANTOS ${ }^{1}$, R. A. BARICCATTI ${ }^{1}$, L. CARDOZO FILHO ${ }^{2}$, C. $_{\text {SILVA }}^{3}$ e E. A. SILVA ${ }^{1}$ \\ ${ }^{1}$ Universidade Estadual do Oeste do Paraná, Centro de Engenharias e Ciências Exatas \\ ${ }^{2}$ Universidade Estadual de Maringá, Departamento de Engenharia Química \\ ${ }^{3}$ Universidade Estadual de Maringá, Departamento de Tecnologia \\ E-mail para contato: edsondeq@ hotmail.com
}

\begin{abstract}
RESUMO - Neste estudo foram investigados os efeitos da temperatura e pressão no rendimento de extração do óleo de crambe (Crambe abyssinica) utilizando como solvente o propano em condições subcríticas. Empregou-se um planejamento fatorial $2^{2}$ completo, com temperatura variando entre 40 e $80{ }^{\circ} \mathrm{C}$, pressão entre 80 e 160 bar, tempo total de extração de 80 minutos e vazão mássica de solvente de $1,6 \times 10^{-3} \mathrm{~kg} \mathrm{~min}^{-1}$. Os resultados mostraram que a temperatura foi a variável mais importante no rendimento de extração para as condições empregadas e o rendimento mais elevado $(32,8 \%)$ foi obtido na condição de maior temperatura e pressão empregadas, $80{ }^{\circ} \mathrm{C}$ e $160 \mathrm{bar}$, respectivamente. Os extratos foram caracterizados quanto ao perfil de ácidos graxos totais e os resultados não revelaram diferenças significativas para as condições experimentais utilizadas, sendo o ácido erúcico o ácido graxo majoritário. Também foi realizada a modelagem matemática da cinética de extração utilizando o modelo de Sovová que representou satisfatoriamente os dados experimentais.
\end{abstract}

\section{INTRODUÇÃO}

O crambe (Crambe abyssinica) é uma oleaginosa da família Brassicaceae, próxima à canola e à mostarda (Desai, 2004; Lazzeri et al., 1994). Suas sementes apresentam elevado teor de óleo, em média 38\% (No, 2011). O óleo de crambe, principal produto da oleaginosa, é rico em ácido erúcico (50-60\%), um ácido graxo monoinsaturado de grande aplicação na indústria farmacêutica, cosmética, de lubrificantes, de plásticos, entre outras (Falasca et al., 2010).

$\mathrm{Na}$ obtenção do óleo de oleaginosas, tradicionalmente emprega-se a extração por prensagem seguida da extração com solvente orgânico, usualmente o hexano. Entretanto, o uso de solventes orgânicos apresenta vários inconvenientes tanto relacionados à qualidade dos produtos obtidos quanto à segurança e impacto ambiental. Além disso, são necessárias etapas posteriores para purificação do óleo (García-Risco et al., 2011).

A extração com fluidos pressurizados torna-se uma alternativa para substituir os métodos convencionais. Trata-se de uma tecnologia limpa, pois, ao final da extração, o solvente é totalmente removido do soluto por uma simples despressurização do sistema e pode ser recuperado. Embora o dióxido de carbono seja o fluido mais empregado neste tipo de extração, o propano subcrítico 
apresenta elevadas taxas de extração quando empregado na obtenção de óleo vegetal, além de se trabalhar com pressões mais baixas, o que se torna uma vantagem para as indústrias de extração de óleos.

Assim, este trabalho visa avaliar o propano em condições subcrítica como fluido de extração do óleo de crambe, bem como investigar os efeitos da temperatura e pressão no rendimento e aplicação do modelo de Sovová na modelagem matemática da cinética de extração.

\section{MATERIAIS E MÉTODOS}

\subsection{Materiais}

Foram utilizadas sementes de crambe (Crambe abyssinica) já secas naturalmente, da variedade FMS Brilhante fornecidas pela Faculdade Assis Gurgacz - FAG, localizada no município de Cascavel - Pr. O material descascado foi triturado e sua distribuição granulométrica classificada por peneiramento de acordo com a série Tyler (12 a 32 mesh), sendo utilizada nos experimentos a fração retida na peneira de 14 mesh. A umidade das sementes $(4,06 \pm 0,02)$ foi determinada por método gravimétrico em estufa e a densidade $\left(1,120 \mathrm{~g} \mathrm{~cm}^{-3}\right)$ por picnometria empregando gás hélio (Micromeritics, modelo Accupyc 1330).

Nas extrações do óleo utilizou-se propano grau analítico 95\% de pureza (Linde Gás) e nas extrações convencionais (Soxhlet) foram utilizados diclorometano 99,5\% (Vetec) e $n$-hexano 99\% (F. Maia). Nas análises dos ácidos graxos foram empregados os reagentes hidróxido de potássio PA (Biotec), metanol 99,8\% (Vetec) e $n$-heptano 99,6\% (Merck).

\subsection{Extração Convencional}

As extrações convencionais do óleo de crambe foram realizadas em conjunto Soxhlet (Laborglas). Aproximadamente $10 \mathrm{~g}$ de sementes foram submetidas à extração exaustiva por 480 minutos com os solventes orgânicos diclorometano e hexano, em suas respectivas temperaturas de ebulição. Ao final da extração o solvente foi evaporado em estufa. Os experimentos foram realizados em duplicata.

\subsection{Extração com Propano Subcrítico}

Os experimentos foram realizados em uma unidade de escala laboratorial O Aparato é constituído de um reservatório para o solvente, uma bomba seringa, dois banhos termostáticos e um extrator de aço inox com capacidade de aproximadamente $58 \mathrm{~cm}^{3}$ (diâmetro de $1,95 \mathrm{~cm}$ e altura de $19,4 \mathrm{~cm}$ ). Realizou-se um planejamento experimental completo $2^{2}$ com ponto central para analisar a influência das variáveis independentes, temperatura e pressão, no rendimento da extração do óleo de crambe. Em cada extração, aproximadamente $30 \mathrm{~g}$ de sementes foram utilizadas para carregar o extrator. Os experimentos foram conduzidos em temperaturas de 40 a $80{ }^{\circ} \mathrm{C}$, pressões de 80 a 160 bar. $\mathrm{Na}$ bomba, o solvente resfriado foi pressurizado em intervalos de pressão de 10 bar até a pressão desejada e então bombeado para extrator, já estabilizado termicamente na temperatura de extração. 
Imediatamente à estabilização da pressão no extrator, a válvula de expansão foi aberta para uma vazão mássica de solvente de $1,6 \times 10^{-3} \mathrm{~kg} \mathrm{~min}^{-1}$. Durante os 80 minutos de extração, o óleo foi coletado em frasco de vidro âmbar e sua massa pesada em intervalos de tempo predefinidos.

\subsection{Modelagem Matemática da Cinética de Extração}

O modelo de Sovová (1994) foi empregado para descrever as curvas cinéticas da extração do óleo com propano em condições subcríticas. De acordo com esse modelo o processo de extração pode ser divido em três etapas: na primeira, a resistência a transferência de massa é a difusão no filme externo, com taxa constante de extração - CER, em que o soluto é de fácil acesso $\left(X_{P}\right)$; na última etapa a taxa de extração é limitada pela difusão na fase sólida, estando o soluto no interior das células com difícil acesso $\left(X_{K}\right)$; e na etapa intermediária estão presentes ambos os mecanismos de transferência de massa (etapa de taxa decrescente de extração - FER). A solução analítica do modelo Sovová é formada pelas Equações 1, 2 e 3.

Para $t<t_{C E R}$ :

$$
m(t)=\dot{m}_{F} Y_{S} t[1-\exp (-Z)]
$$

Para $t_{C E R} \leq t \leq t_{F E R}$ :

$$
m(t)=\dot{m}_{F} Y_{S}\left[t-t_{C E R} \exp \left(\frac{Z Y_{S}}{W X_{0}} \ln \left\{\frac{1}{1-r}\left(\exp \left(\frac{W \dot{m}_{F}}{m_{s}}\right)\left(t_{C E R}-t\right)-r\right)\right\}-Z\right)\right]
$$

Para $t>t_{F E R}$ :

$$
m(t)=m_{s}\left[X_{0}-\frac{Y_{s}}{W} \ln \left\{1+\left(\exp \left(\frac{W X_{0}}{Y_{S}}\right)-1\right) \exp \left(\frac{W \dot{m}_{F}}{m_{s}}\right)\left(t_{C E R}-t\right) r\right\}\right]
$$

Onde:

$$
\begin{aligned}
& Z=\frac{k_{F} a m_{s} \rho_{F}}{\dot{m}_{F} \rho_{S}} \\
& W=\frac{m_{s} k_{S} a}{\dot{m}_{F}(1-\varepsilon)}
\end{aligned}
$$




$$
\begin{aligned}
& t_{C E R}=\frac{(1-r) m_{s} X_{0}}{Y_{S} Z \dot{m}_{F}} \\
& t_{F E R}=t_{C E R}+\frac{m_{s}}{W \dot{m}_{F}} \ln \left[r+(1-r) \exp \left(\frac{W X_{0}}{Y_{S}}\right)\right]
\end{aligned}
$$

Em que $\dot{m}_{F}$ é a vazão mássica de solvente $\left(\mathrm{kg} \mathrm{min}^{-1}\right), Y_{S}$ é a solubilidade do óleo no solvente $\left(\mathrm{g} \mathrm{g}^{-1}\right), t$ é o tempo de extração (min), $X_{0}$ é a concentração inicial do óleo na matriz sólida $\left(\mathrm{g} \mathrm{g}^{-1}\right)$, $m_{S}$ é a massa de sólido isenta de óleo $(\mathrm{g}), r$ é a fração de óleo facilmente acessível $\left(X_{P} / X_{0}\right)$ considerada constante devido ao processo de preparação do ser o mesmo para todo o material, $t_{C E R}$ é o tempo que se leva para o término do primeiro período de extração $(\min ), t_{F E R}$ se refere ao tempo final do segundo período de extração (min), $k_{F} a$ é o coeficiente de transferência de massa no filme externo $\left(\min ^{-1}\right), k_{S} a$ é o coeficiente de transferência de massa no sólido $\left(\min ^{-1}\right), \varepsilon$ é a porosidade do leito, $\rho_{F}$ é a densidade do fluido $\left(\mathrm{kg} \mathrm{m}^{-3}\right), \rho_{S}$ é a densidade do sólido $\left(\mathrm{kg} \mathrm{m}^{-3}\right) Z$ e $W$ são os parâmetros adimensionais do modelo.

O parâmetro $r$ foi ajustado aplicando o método "golden search", com a função objetivo dada pela equação 8:

$$
F=\sum_{i=1}^{n} \sum_{j=1}^{\exp }\left(m_{\text {oil }}^{\text {Calc }}-m_{\text {oil }}^{E x, j}\right)
$$

Os parâmetros $Z$ e $W$ foram determinados com o emprego do método de otimização multivariável Simplex Down-Hill (Nelder \& Mead, 1965) a partir da minimização da função objetivo expressada na Equação 9:

$$
F=\sum_{j=1}^{N}\left(m_{\text {oil }_{j}}^{\text {Calc }}-m_{\text {oil }_{j}}^{\text {Ex }}\right)
$$

Onde: $m_{o i l, j}^{\text {Calc }}$ é a massa de óleo extraída obtida pelo modelo de Sovová (g); $m_{o i l, j}^{E x p}$ é a massa de óleo obtida experimentalmente (g), $n_{-}$exp é o número experimentos e $N$ o número de pontos da curva cinética experimental.

\subsection{Análise dos Ácidos Graxos Totais}

A análise dos ácidos graxos totais presentes no óleo de crambe foi realizada utilizando um cromatógrafo a gás Agilent 7890 acoplado a espectro de massas MS 8990 e equipado com uma coluna capilar (ZBWAX, 30m x 0,25mm x 0,25 $\mu \mathrm{m}$ ) segundo metolodologia de Garcia et al. (2012). 
Com a finalidade de determinar os ácidos graxos totais foi realizada a derivatização do óleo com solução metanólica de KOH $2 \mathrm{~mol} \mathrm{~L}^{-1}$ seguindo a metodologia padrão AOAC Ce 2-66 (1990). A identificação dos componentes presentes no óleo de crambe foi realizada por comparação dos dados do espectro com os apresentados na biblioteca Wiley.

\section{RESULTADOS E DISCUSSÃO}

$\mathrm{Na}$ Tabela 1 são apresentadas as condições experimentais e os resultados das extrações empregando o solvente pressurizado e das extrações convencionais em Soxhlet com $n$-hexano e diclorometano. A solubilidade $\left(Y_{S}\right)$ do óleo no solvente foi determinada pelo método dinâmico através do coeficiente angular da parte linear da curva de extração e o rendimento dado pela razão entre a massa de óleo extraída e a massa de sementes alimentada no extrator.

Tabela 1 - Condições experimentais das extrações e rendimentos

\begin{tabular}{ccccccc}
\hline Solvente & $\begin{array}{c}\text { Condição } \\
\text { experimental }\end{array}$ & $\begin{array}{c}\text { Temperatura } \\
\left({ }^{\circ} \mathrm{C}\right)\end{array}$ & $\begin{array}{c}\text { Pressão } \\
(\text { bar })\end{array}$ & $\begin{array}{c}\rho_{F} \\
\left(\mathrm{~g} \mathrm{~cm}^{-3}\right)\end{array}$ & $\begin{array}{c}Y_{S} \\
\left(\mathrm{~g}_{\text {óleo }} \mathrm{g}_{\text {solvente }}{ }^{-1}\right)\end{array}$ & $\begin{array}{c}\text { Rendimento } \\
(\%)\end{array}$ \\
\hline \multirow{3}{*}{ Propano } & 1 & 40 & 80 & 0,485 & 0,170 & 26,3 \\
Subcrítico & 2 & 40 & 160 & 0,509 & 0,149 & 26,3 \\
& 3 & 80 & 80 & 0,424 & 0,128 & 29,7 \\
$n$-hexano & 4 & 80 & 160 & 0,459 & 0,139 & 32,8 \\
Diclorometano & HEX & 60 & 120 & 0,474 & 0,169 & $29,6^{\mathrm{a}} \pm 0,06$ \\
${ }^{2}$ DCM & - & - & - & & $47,5^{\mathrm{a}} \pm 0,45$ \\
\end{tabular}

${ }^{\mathrm{a}}$ Média da triplicata \pm desvio padrão.

As extrações em Soxhlet comprovaram o elevado teor lipídico nas sementes de crambe, apresentando rendimento de $51 \%$ com a utilização do diclorometano. Nas extrações com propano subcrítico o maior rendimento $(32,8 \%)$ foi obtido na condição de maior temperatura e pressão $\left(80{ }^{\circ} \mathrm{C}\right.$ e 160 bar), sendo que em 80 minutos de extração total, são removidos de 51,6 a 64,3\% do óleo contido nas sementes.

Os teores de ácidos graxos das amostras de óleo de crambe obtidas sob diferentes condições de temperatura e pressão com o solvente propano subcrítico, bem como o óleo extraído de forma convencional com hexano (HEX) e diclorometano (DCM), estão apresentados na Tabela 2. A análise mostrou que não houve diferenças significativas entre os resultados ao nível de significância de $5 \%$. Ainda, verificou-se que os ácidos graxos insaturados representam cerca de $94 \%$ do total de ácidos graxos presentes no óleo, sendo que o majoritário é o ácido erúcico (C22:1), chegando a 59,4\%, seguido do ácido oleico (C18:1) com composição de até 20,17\% e do linoleico (C18:2), até 7,52\%, em concordância com outros trabalhos reportados na literatura (Singh \& Singh, 2010; Lalas et al., 2012). Os ácidos graxos saturados presentes no óleo somam aproximadamente 6\%, sendo que o predominante é o ácido behênico (C22:0), com até 2\% da composição total. 
Tabela 2 - Composição em ácidos graxos totais (\%) encontrada no óleo de crambe extraído sob diferentes condições experimentais (conforme Tabela 1)

\begin{tabular}{lccccccc}
\hline Ácido graxo & 1 & 2 & 3 & 4 & 5 & HEX & DCM \\
\hline Palmítico & 1,74 & 1,75 & 1,72 & 1,87 & 1,87 & 1,72 & 1,79 \\
Palmitoleico & 0,06 & 0,08 & 0,06 & 0,05 & 0,08 & 0,08 & 0,10 \\
Esteárico & 0,93 & 0,85 & 0,84 & 0,85 & 0,94 & 0,89 & 0,93 \\
Oleico & 18,68 & 18,43 & 20,17 & 19,72 & 20,16 & 17,87 & 18,01 \\
cis-vacênico & 0,24 & 0,20 & 0,16 & 0,20 & 0,23 & 0,26 & 0,32 \\
Linoleico & 6,86 & 6,99 & 7,14 & 6,71 & 7,23 & 6,99 & 7,52 \\
Linolênico & 5,14 & 5,05 & 5,71 & 5,08 & 4,92 & 5,37 & 5,05 \\
Araquídico & 1,21 & 1,12 & 0,92 & 1,02 & 1,01 & 1,04 & 1,13 \\
Gadoleico & 3,63 & 3,63 & 3,58 & 3,38 & 3,48 & 3,44 & 3,67 \\
Eicosadienóico & 0,61 & 0,65 & 0,55 & 0,57 & 0,49 & 0,64 & 0,74 \\
Behênico & 1,75 & 1,91 & 1,91 & 1,58 & 1,62 & 1,77 & 2,02 \\
Erúcico & 58,64 & 58,89 & 56,72 & 58,53 & 57,42 & 59,38 & 58,49 \\
Lignocérico & 0,33 & 0,40 & 0,35 & 0,29 & 0,43 & 0,37 & 0,39 \\
Total & 99,82 & 99,95 & 99,83 & 99,85 & 99,88 & 99,82 & 100,00 \\
\hline
\end{tabular}

Os resultados das extrações com propano subcrítico indicam que a temperatura apresenta um efeito positivo na extração, ou seja, o aumento desta variável proporciona maiores rendimentos. Para a variável pressão verifica-se um efeito positivo apenas para os experimentos realizados na maior temperatura do planejamento $\left(80{ }^{\circ} \mathrm{C}\right)$, o que se se deve à temperatura empregada estar mais próxima da temperatura crítica do propano, resultando na melhora do poder de solvatação do solvente devido ao aumento em sua densidade. Efeito similar foi encontrado por Pederssetti et al. (2011); Nimet et al. (2011) e Corso et al. (2010), na extração com propano subcrítico dos óleos de canola, girassol e gergelim, respectivamente.

Na modelagem matemática da cinética de extração foram utilizados os seguintes parâmetros: concentração inicial de óleo de $0,488 \mathrm{~g}_{\text {óleo }} \mathrm{g}_{\text {sólido }}{ }^{-1}$ (dada pela razão entre a massa de óleo nas sementes e a massa de sólido inerte), densidade do sólido de $1,120 \mathrm{~g} \mathrm{~cm}^{-3}$, densidade do leito de $0,343 \mathrm{~g} \mathrm{~cm}^{-3}$, porosidade de 0,684 , massa de material inerte de $19,9 \mathrm{~g}$, vazão mássica de solvente de $1,6 \times 10^{-3} \mathrm{~kg}$ $\mathrm{min}^{-1}$, densidade do solvente e solubilidade do óleo no solvente de acordo com a Tabela 1.

Na Tabela 3 são apresentados os parâmetros ajustados do modelo de Sovová ( $Z, W$ e $r$ ). Devido à dificuldade em extrair o óleo contido no interior das células intactas, considera-se a última etapa de extração como sendo a limitante do processo, sendo o rendimento final função da taxa de transferência de massa. $\mathrm{O}$ maior valor de $k_{S a}$ foi obtido na condição de maior temperatura e pressão $\left(80{ }^{\circ} \mathrm{C}\right.$ e $160 \mathrm{bar}$ ), evidenciando o maior rendimento obtido neste experimento. Ainda, pode-se relacionar este parâmetro aos efeitos da temperatura e pressão citados anteriormente, sendo este maior quanto maior a temperatura empregada. 
Tabela 3 - Parâmetros ajustados do modelo de Sovová para as extrações com propano subcrítico

\begin{tabular}{cccccccccc}
\hline $\begin{array}{c}\mathrm{T} \\
\left({ }^{\circ} \mathrm{C}\right)\end{array}$ & $\begin{array}{c}\mathrm{P} \\
(\mathrm{bar})\end{array}$ & $Z$ & $W$ & $r$ & $\begin{array}{c}t_{C E R} \\
(\mathrm{~min})\end{array}$ & $\begin{array}{c}t_{F E R} \\
(\mathrm{~min})\end{array}$ & $\begin{array}{c}k_{F a} \\
\left(\mathrm{~min}^{-1}\right)\end{array}$ & $\begin{array}{c}k_{S a} \\
\left(\mathrm{~min}^{-1}\right)\end{array}$ & $\begin{array}{c}\text { Erro médio } \\
(\%)\end{array}$ \\
\hline 40 & 80 & 2,146 & 0,297 & 0,670 & 12,166 & 41,534 & 0,111 & $6,924 \times 10^{-3}$ & 5,191 \\
40 & 160 & 4,312 & 0,246 & 0,670 & 6,276 & 36,550 & 0,235 & $6,298 \times 10^{-3}$ & 1,660 \\
80 & 80 & 11,875 & 0,401 & 0,670 & 2,620 & 39,948 & 0,788 & $1,040 \times 10^{-2}$ & 3,507 \\
80 & 160 & 8,769 & 0,583 & 0,670 & 3,206 & 38,200 & 0,549 & $1,541 \times 10^{-2}$ & 3,513 \\
60 & 120 & 9,303 & 0,365 & 0,670 & 2,567 & 30,012 & 0,546 & $9,336 \times 10^{-3}$ & 5,704 \\
\hline
\end{tabular}

As curvas cinéticas experimentais de extração ajustadas com o modelo de Sovová são apresentadas na Figura 1. O modelo apresentou bom ajuste aos dados experimentais $\left(R^{2}>0,99\right)$ para todas as condições empregadas, com erro médio absoluto máximo de 5,704\%.

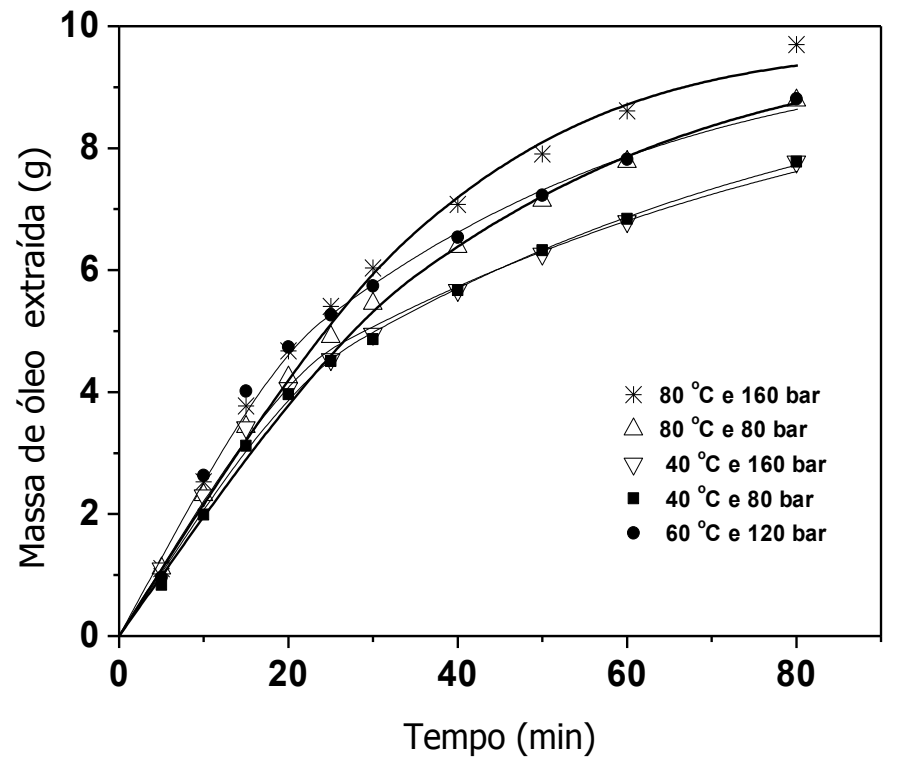

Figura 1 - Cinéticas experimentais de extração com ajuste do modelo de Sovová.

\section{CONCLUSÕES}

As extrações do óleo de crambe com propano subcrítico proporcionaram elevados rendimentos em 80 minutos. A melhor condição foi a de maior pressão e temperatura $\left(80{ }^{\circ} \mathrm{C}\right.$ e 160 bar $)$, na qual foi obtido $32,8 \%$ de rendimento. Para as condições experimentais empregadas, somente a temperatura apresentou influência no rendimento da extração. O modelo cinético de Sovová se ajustou adequadamente aos dados experimentais. 


\section{REFERÊNCIAS}

CORSO, M. P.; SILVA, E. A.; KLEN, M. R. F.; CARDOZO-FILHO, L.; SANTOS, J. N.; FREITAS, L. S.; DARIVA, C. Extraction of sesame seed (Sesamun indicum L.) oil using compressed propane and supercritical carbon dioxide. J. Supercrit. Fluids, v. 52, p. 56-61, 2010.

DESAI, B. B. Seeds handbook biology production processing and storage. New York: Marcel Dekker, 2004.

FALASCA, S. L.; FlORES, N.; LAMAS, M. C.; CARBALlO, S. M.; ANSCHAU, A. Crambe abyssinica: an almost unknown crop with a promissory future to produce biodiesel in Argentina. Int. J. Hydrog. Energy, v. 35, p. 5808-5812, 2010.

GARCIA, V. A. S.; CABRAL, V. F.; ZANOElO, E. F.; SIlVA, C.; CARDOZO-FILHO, L. Extraction of Mucuna seed oil using supercritical carbon dioxide to increase the concentration of 1Dopa in the defatted meal. J. Supercrit. Fluids, v. 69, p. 75-81, 2012.

GARCÍA-RISCO, M. R. G.; VICENTE, G.; REGLERO, G.; FORNARI, T. Fractionation of thyme (Thymus vulgaris L.) by supercritical fluid extraction and chromatography. J. Supercrit. Fluids, v. 55, p. 949-954, 2011.

LALAS, S.; GORTZI, O; ATHANASIADIS, V. Full Characterisation of Crambe abyssinica Hochst. Seed Oil. J. Am. Oil Chem. Soc., v. 89, p. 2253 -2258, 2012.

LAZZERI, L.; LEONI, O.; CONTE, L.; PALMIERI, S. Some technological characteristics and potential uses of Crambe abyssinica products. Ind. Crop. Prod., v.3, p. 103-112, 1994.

NELDER, J. A.; MEAD, R. A simplex method for function minimization. Comput. J., v. 7, p. 308$313,1965$.

NIMET, G.; SILVA, E. A.; PALÚ, F.; DARIVA, C.; FREITAS, L. S.; NETO, A. M.; CARDOZOFILHO, L. Extraction of sunflower (Heliantus annuus L.) oil with supercritical $\mathrm{CO}_{2}$ and subcritical propane: Experimental and modeling. Chem. Eng. J., v. 168, p. 262 - 268, 2011.

NO, S. Y. Inedible vegetable oils and their derivatives for alternative diesel fuels in CI engines: a review. Renew. Sust. Energ. Rev., v. 15, p. 131-149, 2011.

PEDERSSETTI, M. M.; PAlÚ, F.; SIlVA, E. A.; ROHLING, J. H.; CARDOZO-FILHO, L.; DARIVA, C. Extraction of Canola Seed (Brassica napus) Oil using Compressed Propane and Supercritical Carbon Dioxide. J. Food Eng., v. 102, p. 189-196, 2010.

SINGH, S. P.; SINGH, D. Biodiesel production through de use of different sources and characterization of oils and their esters as the substitute of diesel: a review. Renew. Sust. Energ. Rev., v. 14, p. 200-216, 2010.

SOVOVÁ, H. Rate of Vegetable Oil Extraction with Supercritical $\mathrm{CO}_{2}-\mathrm{I}$ and II Modelling of Extraction Curves. Chem. Eng. Sci., v. 49, n. 3, p. 409,1994.

WALKER, R.E. Official methods and recommended practices of the American Oil Chemists' Society (Method AOCS Ce 2-66) (4th). Champaign: American Oil Chemists Society, 1990. 